\title{
Cytokine Impregnated Biomatrix: A New Tool to Study Multi-Wall Carbon Nanotubes Effects on Invertebrate Immune Cells
}

\author{
Rossana Girardello', Francesco Drago², Magda de Eguileor ${ }^{1}$, Roberto Valvassori ${ }^{1}$, Jacopo Vizioli², Gianluca Tettamanti ${ }^{1}$ and Annalisa \\ Grimaldi* $^{1 *}$ \\ ${ }^{1}$ Department of Biotechnology and Life Sciences, University of Insubria, Via J. H. Dunant 3, 21100 Varese, Italy \\ 2Inserm U1192, Laboratoire de Protéomique, Réponse Inflammatoire, Spectrométrie de Masse (PRISM), Université Lille 1, Cité Scientifique, 59655 Villeneuve D’Ascq, \\ France
}

\begin{abstract}
The novel features of engineered nanoparticles, such as multi-wall carbon nanotubes (MWCNTs) are impressive and attractive for technology, however they dissolved in water and accumulate in soils through the application of sewage sludge, accidental spills, and deposition from the air, agrochemicals or soil remediation. Given that several studies have revealed that chronic exposure to these nanomaterials products through the ingestion of drinking water, inhalation and dermal contact may harbour potential risks to human health, risk assessment of this nanomaterials in the aquatic environment are becoming essential. Here we propose a freshwater invertebrate, the leech Hirudo medicinalis, as a model to assess the effects MWCNTs on the immune system by means of in vivo and in vitro experiments. For this study, we used a consolidated experimental approach based on injection in the body wall of the leech of the biomatrice Matrigel (MG), added with a specific macrophage chemoattractant, the cytokine Allograft inflammatory factor-1 (AIF-1) and/or with MWCNTs. MG sponges analysis show the presence of a larger number of cells positive for both CD68 and HmAIF-1, specific monocyte-macrophage markers. Ultrastructural analysis suggests that MWCNTs may be internalized by phagocytosis but they seem also to be able to pierce cell membranes during cells migration.
\end{abstract}

Cells extracted from MG were also used for in vitro treatment with MWCNTs at different concentration $(2.5,5,10$, 25,50 and $100 \mu \mathrm{g} / \mathrm{ml}$ ) for $24 \mathrm{~h}$ to study cell morphology changes and production of amyloid fibrils in order to encapsulate the foreign bodies. Our results, not only confirm the ability of MWCNTs in inducing a potent inflammatory response, but highlight rapid colorimetric assays that can be successfully used as sensitive tools for aquatic pollution biomonitoring.

Keywords: Multi-wall carbon nanotubes (MWCNTs); Hirudo medicinalis; Macrophages; Matrigel; Innate immune response; Amyloid fibrils

\section{Introduction}

The rapid development of nanotechnology and nanoscience during the last decade has led to the discovery of nanomaterials such as carbon nanotubes, classified into single-walled (SWCNTs) and multiwalled (MWCNTs) carbon nanotubes, which have several potential applications. The former are mainly used in biology and medicine [1], while the latter are widely used in industry, and their applications are increasing constantly in particular in waste water treatment [2]. Due to their massive production and in the light of the most recent studies, which emphasize the potential toxicity of nanotubes, it is more than ever necessary to take into account their impact on the environment [3]. Aquatic ecosystems seem to be particularly susceptible to contamination by MWCNTs and other pollutants with harmful consequences for the organisms that inhabit them. Several studies, in fact, demonstrate MWCNTs toxicity and bio-persistence within tissues and cells [4-6]. The bioaccumulation of MWCNTs in aquatic animals may as well represent a risk to humans, who may be exposed to this nanomaterial through many pathways, such as inhalation, injection, penetration but also ingestion [7]. In particular several studies revealed that the physical dimension of MWCNTs are critical factors in mesothelial injury and carcinogenesis [8] and are associated with phagocytosis [9] that leads to the production of pro-inflammatory cytokines and reactive oxygen species (ROS) [10]. Although the literature abounds with studies on MWCNTs toxicity, the existing toxicological data are still fragmentary and their biological interactions with cells, proteins and tissues have not yet been fully understood [3].

The aim of this work is to develop and optimize approaches to suggest an invertebrate animal model able to give rapid and sensitive responses upon the presence of pollutions in water, such as nanoparticles, even if at low concentration. Immune system of organisms represents a sensitive physiological indicator that may be affected even at low concentrations of nanomaterial exposure; however the animal models usually employed in ecotoxicological studies are not suitable due to their immune responses strictly linked to their bauplan.

The model organism chosen in this study is the leech Hirudo medicinalis. This aquatic invertebrate is suitable for experimental manipulation, economic, easily treated and without significant ethical considerations related to use and regulatory restrictions. Leech is a new interesting animal model for several reasons: i) its immune response processes includes the same steps described for vertebrate [11-15], involving similar cellular mechanisms and key molecules that play pivotal roles for guiding and regulating the hematopoietic cells activation and differentiation, revealing a conserved regulation immune response processes; ii) any response evoked by different stimuli are activated within a short period of time $(6,24$ hours) and clear and easily detectable due to their small size and anatomical

*Corresponding author: Annalisa Grimaldi, Department of Biotechnology and Life Sciences, University of Insubria, Via J. H. Dunant, 3, 21100 Varese, Italy, Tel: +390332421325; Fax: +390332421300; E-mail: annalisa.grimaldi@uninsubria.it

Received June 24, 2015; Accepted August 16, 2015; Published October 01, 2015

Citation: Girardello R, Drago F, de Eguileor M, Valvassori R, Vizioli J, et al (2015) Cytokine Impregnated Biomatrix: A New Tool to Study Multi-Wall Carbon Nanotubes Effects on Invertebrate Immune Cells. J Nanomed Nanotechnol 6: 323 doi:10.4172/2157-7439.1000323

Copyright: (c) 2015 Girardello R, et al. This is an open-access article distributed under the terms of the Creative Commons Attribution License, which permits unrestricted use, distribution, and reproduction in any medium, provided the original author and source are credited. 
simplicity; iii) it shows an added value related to a novel developed assay based on the use of a biomatrix (MATRIGEL) [16-18] to obtain in vitro expansion of macrophages primary leech cells implicated in immune response process that could be used to test the effect of the nanomaterial on immune cells.

Our combined experimental approaches, based on high sensitive inflammatory response and rapid colorimetric tests, can highlight adverse effects of MWCNTs on immune system cells, even at low concentration, and could be used as quick sensitive tools for aquatic pollution bio-monitoring.

\section{Materials and Methods}

\section{Animals and treatment}

Leeches (H. medicinalis, Annelida, Hirudinea, from Ricarimpex, Eysines, France) measuring $10 \mathrm{~cm}$ were kept in water at $19-20^{\circ} \mathrm{C}$ in aerated tanks. Animals were fed weekly with calf blood. Animals were randomly divided into separate experimental groups according to different protocols and treatments. Each treatment was performed at the level of the $20^{\text {th }}$ metamere. Before each experiment, leeches were anaesthetized with a $10 \%$ ethanol solution.

Group 1: Leeches injected with $300 \mu \mathrm{l}$ of liquid MG (an extract of the murine Engelbreth-Holm-Swarm (EHS) tumor produced as previous described [19]) served as controls.

Group 2: Leeches (five animals/condition) injected with $300 \mu$ of liquid MG supplemented with 100, 150 and $300 \mathrm{ng}$ of the recombinant protein $\mathrm{rHmAIF}-1$ (kindly donated by Jacopo Vizioli and Francesco Drago, University of Lille, France), were used to selectively isolate the macrophages cells migrating under the influence of HmAIF-1. The best concentration of $\mathrm{rHmAIF-1}$ required to induce significant cell migration was $300 \mathrm{ng}$ and was used for the next experiments.

Group 3: Leeches injected with $300 \mu \mathrm{l}$ of liquid MG supplemented with $20 \mu \mathrm{g}$ of Multi-walled commercially available and industrially employed Carbon Nanotubes (MWCNTs), NANOCYL ${ }^{\mathrm{TM}}$ NC7000 (Belgium NANOCYL, Sambreville; average $9.5 \mathrm{~nm}$ diameter by 1.5 $\mu \mathrm{m}$ mean length with surface area of $250-300 \mathrm{~m}^{2} / \mathrm{g}$, not functionalized, manufactured by catalytic carbon vapour deposition (CCVD) process, with a purity of $90 \%$ ). For these experiments, the pristine MWCNTs were used directly without any chemical processing before use. This concentration of MWCNTs was necessary to obtain visible aggregates in the Matrigel pellet.

Group 4: Leeches injected with $300 \mu$ of liquid MG supplemented with $300 \mathrm{ng}$ rHmAIF-1 and $20 \mu \mathrm{g}$ of MWCNTs.

Animals were sacrificed after 1 week. MG implants were removed from the animal and processed in different ways depending on the type of analyses.

\section{Transmission electron microscopy (TEM)}

MG implants for routine transmission electron microscopy (TEM) were fixed in $2 \%$ glutaraldehyde in $0.1 \mathrm{M}$ sodium cacodylate buffer $(\mathrm{pH}$ 7,2 ) for 1 hour at room temperature. Specimens were washed in the same buffer and then post-fixed for $1 \mathrm{~h}$ with $1 \%$ osmium tetroxide in cacodylate buffer at room temperature. After standard dehydration in ethanol series, specimens were treated with propylene oxide/ EponAraldite (1:1) for 1 hour and then embedded in an Epon-Araldite 812 mixture. Sections were obtained with a Reichert Ultracut $\mathrm{S}$ ultratome (Leica, Nussloch, Germany). Thin sections (80-90 nm) were stained by uranyl acetate and lead citrate and observed with a Jeol 1010 EX electron microscope (Jeol, Tokyo, Japan). Images were acquired with digital camera Morada, Olympus (Tokyo, Japan).

\section{Colorimetric and indirect immunofluorescence staining}

MG implants were embedded in Polyfreeze tissue freezing medium (Polysciences, Eppelheim, Germany) and immediately frozen in liquid nitrogen. Cryosections $(7 \mu \mathrm{m})$ were obtained with a Leica CM1850 cryotome and slides were immediately used or stored at $-20^{\circ} \mathrm{C}$.

For colorimetric assays, cryosections were immersed in distilled water and incubated with May Grunwald Giemsa differential staining (Bio Optica, Milano, Italy), which permits identification of hematopoietic cells based upon their cytoplasmic $\mathrm{pH}$ properties.

For Thioflavin-S method, samples were treated with Mayer's hemalum for 2 minutes. After washings with water samples were stained with $1 \%$ Thioflavin-S in aqueous solution. After further washing, they were treated with $1 \%$ acetic acid for 20 minutes. This method stains the amyloid fibrils in green/yellow fluorescence.

For indirect immunofluorescence, samples washed with PBS were pre-incubated for 30 min with PBS containing $2 \%$ bovine serum albumin (BSA) before the primary antibody incubation for 1 hour at room temperature. The primary antibodies (diluted 1:200) used were: rabbit anti-human CD45 (GenScript, USA), which reacts with leech hematopoietic precursors cells, rabbit anti-human CD68 (Santa Cruz Biotechnology, USA), which reacts with leech macrophages, as previously demonstrated [14], and rabbit anti-Hm-AIF-1 (kindly donated by Jacopo Vizioli and Francesco Drago, University of Lille). The washed specimens were incubated for $1 \mathrm{~h}$ at room temperature with an anti-rabbit secondary antibody (Jackson Immuno Research Laboratories, USA) Cy3 conjugated (dilution 1:200). After further washing with PBS, cryosections were incubated for 10 minutes with the nuclear marker DAPI (4',6-diamidino-2-phenylindole). The slides were mounted in Citifluor (Citifluor Ltd, London, UK) with coverslips and observed by light and fluorescence microscopy Nikon Eclipse Ni (Nikon, Japan).

The staining was visualized using excitation/emission filters of 490/525 nm for Thioflavin-S, 550/580nm for Cy3 and 360/420 nm for DAPI. Data were recorded with Nikon Digital Sight DS-SM (Nikon, Japan) digital camera. Images were combined with Adobe Photoshop (Adobe Systems, Inc.). In control samples, primary antibodies were omitted and sections were treated with BSA-containing PBS.

\section{In vitro matrigel assays}

After 1 week in vivo (corresponding to a suitable cell concentration for seeding) rHmAIF-1 supplemented MG implants were harvested and cultured. Each implant was minced in small pieces using sterilized razor blades and plated in wells of $60 \mathrm{~mm}$ in diameter in DMEM medium (Celbio, Milan, Italy) modified by dilution (1:4) to reach isoosmolality and supplemented with $1 \%$ glutamine, $10 \%$ fetal bovine serum and $1 \%$ gentamicin [16]. Cells were maintained at $20^{\circ} \mathrm{C}$ and histologically and immunocytochemically examined 1 days and 1 week after seeding.

All cultures were performed in triplicate and scored at 1 week from seeding with an inverted Olympus microscope (Tokyo, Japan). Data were recorded with a DS-5M-L1 digital camera system (Nikon).

\section{Assessment of cell viability with trypan blue}

Cell viability was assessed using the vital dye Trypan blue, which is incorporated only in dying or dead cells. The cells were incubated at 
Citation: Girardello R, Drago F, de Eguileor M, Valvassori R, Vizioli J, et al. (2015) Cytokine Impregnated Biomatrix: A New Tool to Study Multi-Wall Carbon Nanotubes Effects on Invertebrate Immune Cells. J Nanomed Nanotechnol 6: 323. doi:10.4172/2157-7439.1000323

Page 3 of 8

room temperature for 5 min with $0.4 \%$ trypan blue solution (diluted 1:1 in basal medium) and directly observed using an inverted optical microscope (Olympus).

\section{In vitro MWCNTs treatment}

Cells were cultured on a round coverslip in 24 wells plate for 1 week before MWCNTs treatment. MWCNTs powder was weighed resuspended in culture medium and then sonicated 15 min for 2 cycles in an ultrasonic bath (starsonic 35, Liarre, Italy) to avoid particles aggregation. MWCNTs were administrated at 2.5, 5, 10, 25, 50 and 100 $\mu \mathrm{g} / \mathrm{ml}$ for $24 \mathrm{~h}$. Particle exposure concentrations were chosen upon assessment of existing literature concerning exposure of vertebrate macrophages cell lines to MWCNTs and SWCNTs [3]. Cells were then fixed with paraformaldehyde $3 \%$ for 10 minutes and washed in PBS before proceeding with immunocytochemical and colorimetric assays described above.

\section{Statistical analysis}

Cells extracted from matrigel pellet were plated out in $60 \mathrm{~mm}$ diameter at a density of 1000 cells/well and counted after one week from seeding, using the Image J software package. Five fields for each time lapse ( 1 day and 1 week) were analyzed and the number of counted cells is referred to the total area of the well. Statistical significance was assessed by an unpaired Student's $t$ test using Origin 5.0 software (Microcal).

\section{Results}

\section{Morphological, immunocytochemical and colorimetric characterization of cells infiltrating the MG sponge}

Light and trasmission electron microscopy: First, since MG can be supplemented with different concentrations of cytokines, we sought the best rHmAIF-1 concentration for the macrophages tissue cells recruitment in the biomatrix. We supplemented $300 \mu \mathrm{l}$ of MG with 100, 150 (data not shown) and $300 \mathrm{ng}$ of rHmAIF1 and the MG pellets, formed following inoculation, were recovered and processed for standard histology one week after injection. While cellular infiltrates were not observed in control MG samples (Figure 1A), the number of cells migrating in the biomatrix increased in relation to the rHmAIF-1 concentration with the highest cell density obtained with $300 \mathrm{ng}$ of rHmAIF-1 in the MG (Figure 1B). In order to assess the interaction between MWCNTs and cells migrated in the MG pellet, we used the biopolymer matrigel supplemented with both $\mathrm{rHmAIF-1}$ and MWCNTs. Numerous migrated cells were visible in the MG sponge,

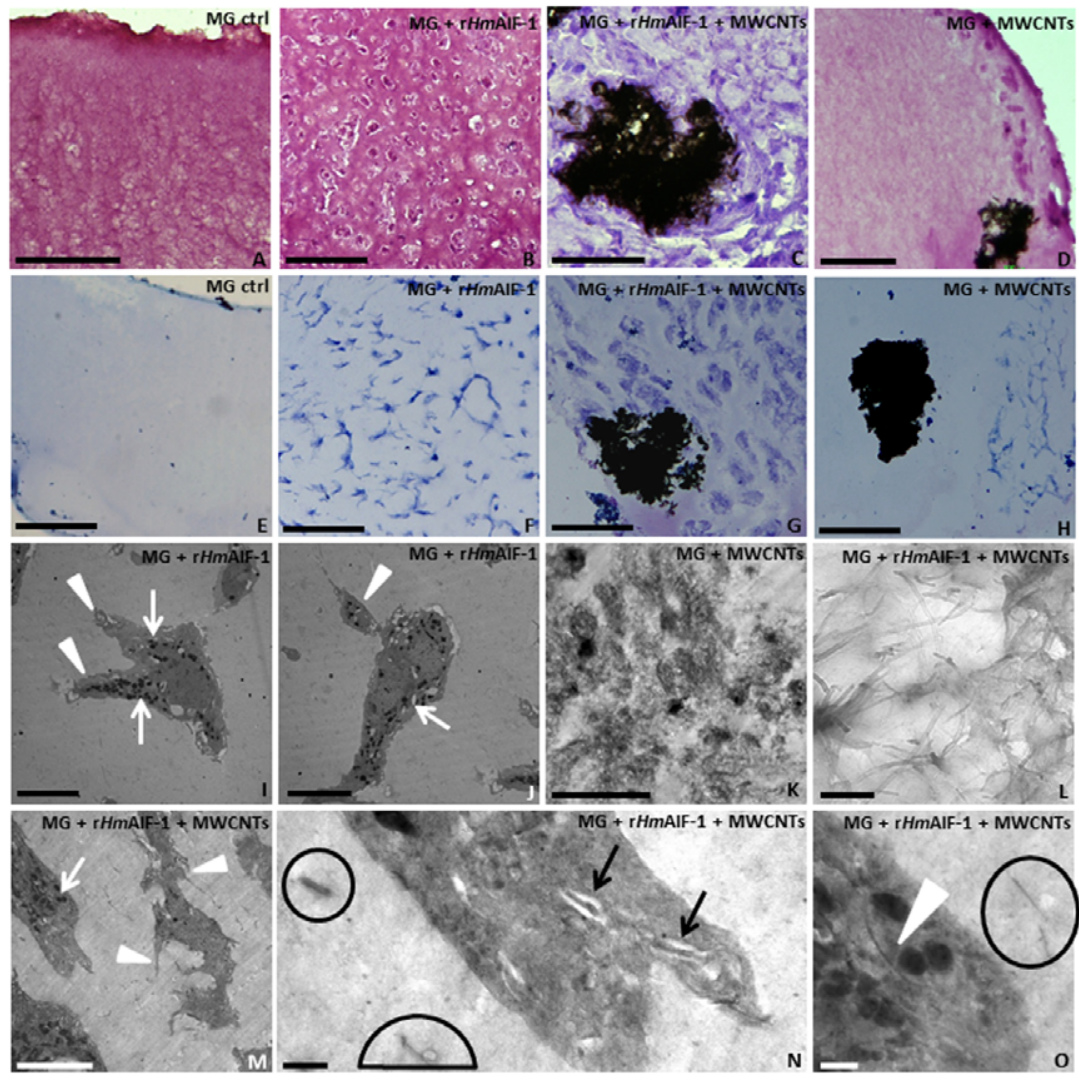

Figure 1: Morphological and colorimetric analyses of cells infiltrating the MG removed from the animal 1 week after injection. (A-H) Appearance under the light microscope of the cells infiltrating the gels without (A, E) and with the rHm AlF-1 (B, F). The rHmAlF-1 recruits a large number of cells (B) positively stained by May GrunwaldGiemsa method (E, F). (C, D, G, H) in MG pellet supplemented with both MWCNTs and rHmAIF-1 (C, G) or only with MWCNTs (D, H), migrating cells positive for GrunwaldGiemsa staining $(\mathrm{G}, \mathrm{H})$ are visible dispersed in the biomatrix and surrounding the MWCNTs aggregates (arrowheads). (I-O) TEM images. (I, J, M-O) Detail of cells infiltrating the MG pellets characterized by a cytoplasm with numerous highly electron-dense granules (arrows), pseudopodia (arrowheads in I, J) and lamellipodia (arrowheads in M). (K, L, N, O) Detail of MWCNTs dispersed or grouped in aggregates differently sized inside the MG sponge $(\mathrm{K}, \mathrm{L}$, encircled in $\mathrm{N}, \mathrm{O}$ ) or in intracellular vesicles (arrows in $\mathrm{N}$ ) and freely dispersed in the cytoplasm (arrowhead in O). Bars in A-H: $50 \mu \mathrm{m}$; Bars in I-K, M: $5 \mu \mathrm{m}$; Bars in L: $200 \mathrm{~nm}$; Bars in N, O: $500 \mathrm{~nm}$. 
Citation: Girardello R, Drago F, de Eguileor M, Valvassori R, Vizioli J, et al. (2015) Cytokine Impregnated Biomatrix: A New Tool to Study Multi-Wall Carbon Nanotubes Effects on Invertebrate Immune Cells. J Nanomed Nanotechnol 6: 323. doi:10.4172/2157-7439.1000323

Page 4 of 8

most forming a clot around the large MWCNTs aggregates (Figure 1C). Migrating cells, even if in a reduced number, was present in the MG pellet supplemented only with MWCNTs (Figure 1D). Cells coloured with the May Grunwald Giemsa differential staining showed dark blue nuclei typical of monocyte and macrophages cell lines (Figure 1E-1H).

Ultrastructural analysis at TEM showed that in MG supplemented only with rHmAIF-1 cells appear either round or with an irregular shape with a cytoplasm rich in electron-dense granules, with a pronounced migratory phenotype showing pseudopodia and an active degradation of the surrounding matrix (Figure 1I and 1J). Cells migrated in MG pellet supplemented with MWCNTs, visible dispersed throughout the MG sponge or grouped in aggregates (Figure $1 \mathrm{~K}$ and 1L), showed increased membrane ruffling and lamellipodia associated with phagocytosis (Figure 1M). Moreover, particulate acquisition was evident as engulfment of particles settled in vesicles or in the cytoplasm (Figure $1 \mathrm{~N}$ and $1 \mathrm{O}$ ).

Immunocytochemical characterization: In order to better characterize the cells infiltrating the $\mathrm{MG}$ and surrounding the MWCNTs aggregates, the Matrigel pellets were removed from the leech after one week from injection and sections of the biopolymer were immunostained with antibodies to macrophage cell markers. The cells infiltrating the Matrigel sponge, either supplemented with only the rHmAIF-1 protein, with both rHmAIF-1 and MWCNTs or with only MWCNTs, were strongly positive for the antibodies anti-CD68
(Figure 2A-2C) and anti-HmAIF-1 (Figure 2D-2F). In particular, in the MG containing MWCNTs, numerous infiltrating CD68 ${ }^{+} / \mathrm{HmAIF-1}{ }^{+}$ macrophages reached and surrounded the MWCNTs aggregates (Figure 2 B, 2C, 2E and 2F). No signal was detected in negative control experiments where primary antibodies were omitted (Figure $2 \mathrm{G}$ ).

Thioflavin-S staining for amyloid fibrils detection: Since recently has been demonstrated that MWCNTs aggregates induce macrophage recruitment, tissue inflammation and amyloid deposition in mice [20], we performed a Thioflavin-S staining on MWCNTs supplemented MG sponges to reveal the presence of amyloid fibrillar material. Thioflavin-S positivity was found in the macrophages surrounding the aggregates of MWCNTs, indicating that these cells were implicated in the production and deposition of amyloid fibrils (Figure 2H and 2I).

\section{Cultured Cells and MWCNTs in vitro treatment}

In order to obtain primary cultures of leech macrophages cells, the MG polymers supplemented with $\mathrm{rHmAIF}-1$ was removed from the leeches after 1 week in vivo and the cells placed in culture. Starting from day 1 after seeding, cultured cells were present as clusters (Figure $3 \mathrm{~A}$ and $3 \mathrm{~B}$ ). One week after seeding, cultures were formed by an increased number of cells (Figure $3 \mathrm{C}$ ). To determine the growth and viability characteristics of the cultured cells, the cells from the matrigel polymers that had been removed from the leeches after 1 week in vivo, were plated at a density of 1000 cells for each well, and the number of
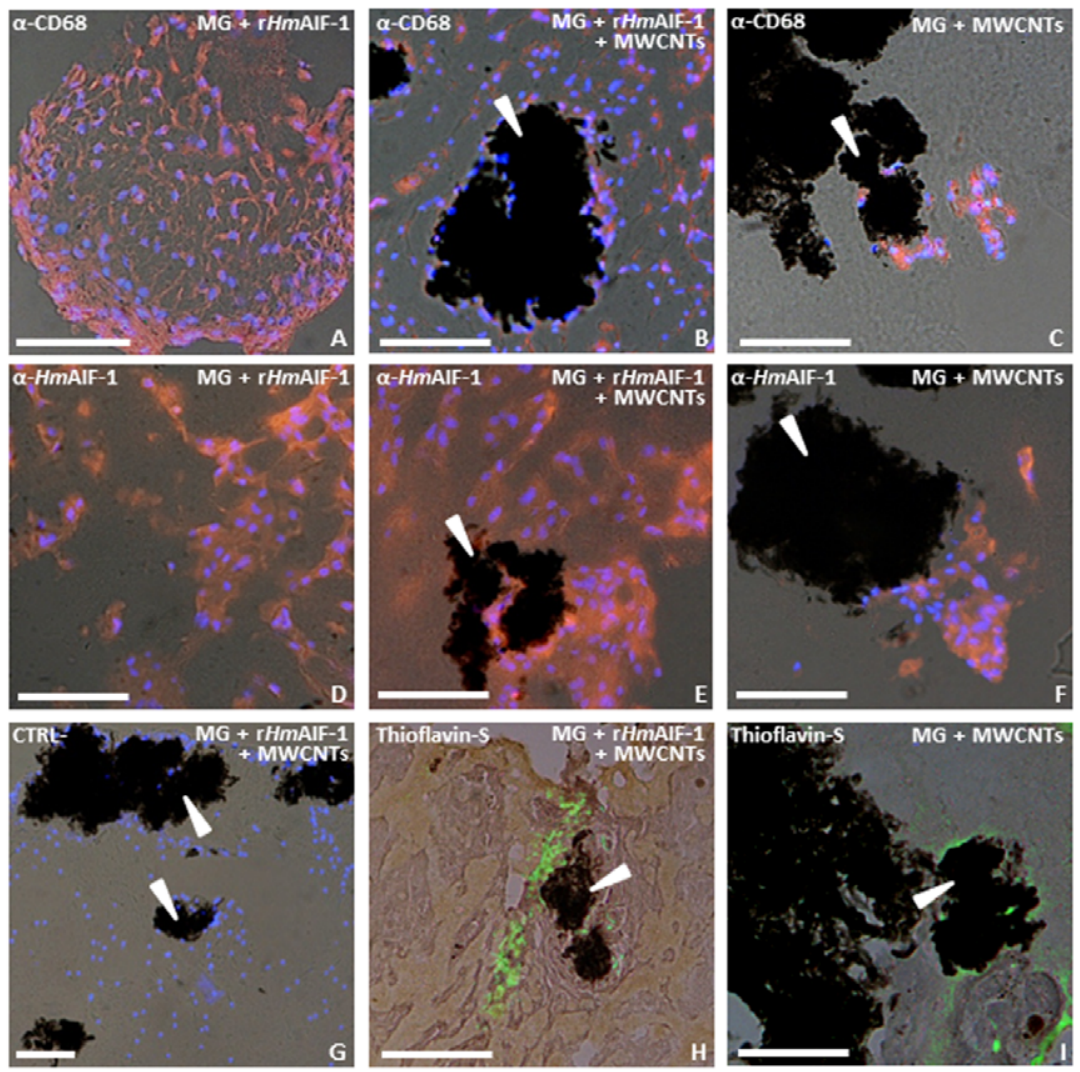

Figure 2: Immunocyto chemical characterization of macrophage cells recruited into the matrigel sponges by $\mathrm{rHmAIF-1}$ and MWCNTs and removed 1 week after injection. Combined fluorescence/transmission images of MG implants cryosections shows that the numerous cells a-CD68 (red in A-C) and $\alpha-H m A I F-1$ (red in $\mathrm{D}-\mathrm{F}$ ) infiltrate the biomatrix and surround the MWCNTs aggregates (arrowheads in B, C, E, F). Nuclei are counterstained with DAPI (blue). (G) Negative control. $(\mathrm{H}-\mathrm{I})$ Thioflavin-S staining recognizes amyloid structures (yellow in $\mathrm{H}, \mathrm{I}$ ) associated to macrophages infiltrating the MWCNTs supplemented MG sponge or forming a scaffold around the MWCNTs aggregates (arrowheads in $\mathrm{H}, \mathrm{I}$ ). Bars in A-I: $50 \mu \mathrm{m}$. 
Citation: Girardello R, Drago F, de Eguileor M, Valvassori R, Vizioli J, et al. (2015) Cytokine Impregnated Biomatrix: A New Tool to Study Multi-Wall Carbon Nanotubes Effects on Invertebrate Immune Cells. J Nanomed Nanotechnol 6: 323. doi:10.4172/2157-7439.1000323

Page 5 of 8
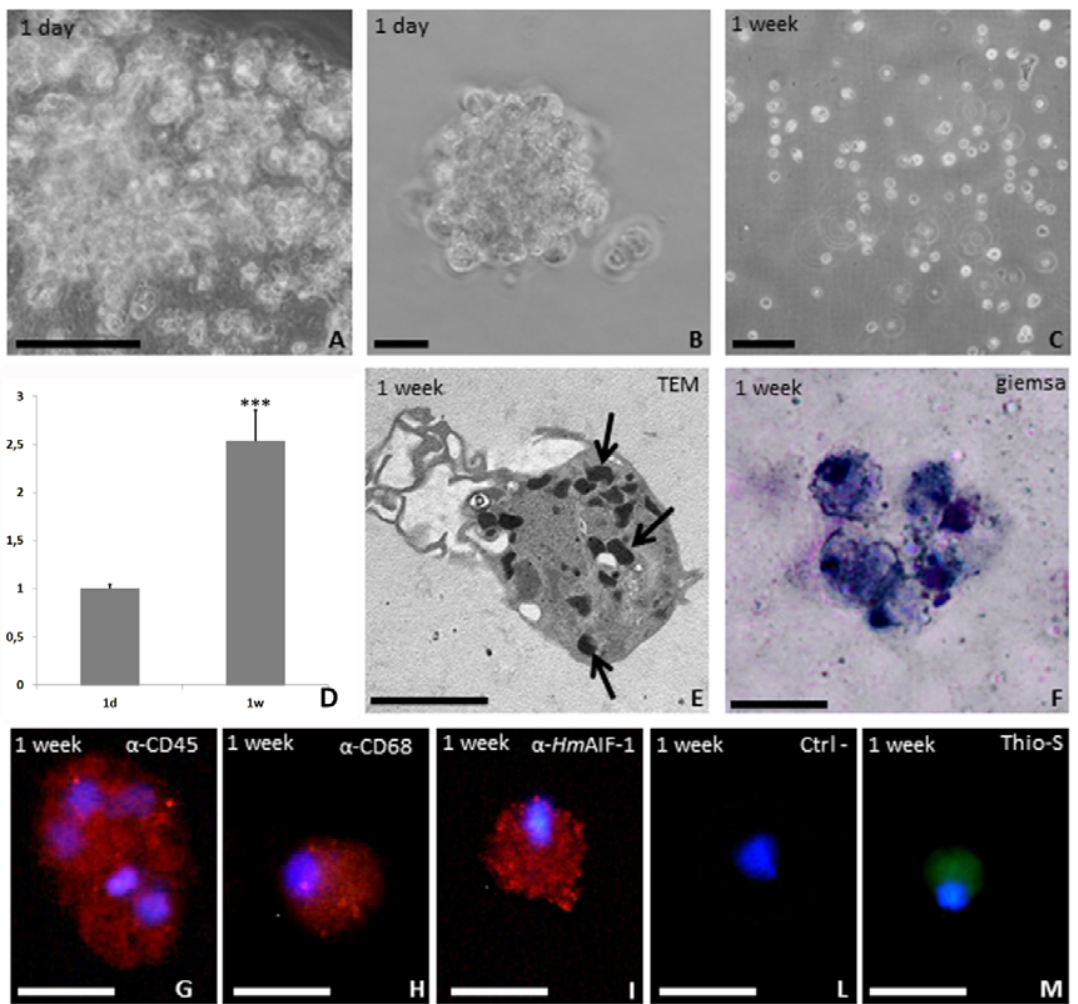

Figure 3: Culture of cells recruited into the matrigel sponges by rHm AlF-1. After 1 week in vivo the MG was removed and the cells infiltrating the matrigel sponge were plated out. Phase contrast image of cultured cells 1 day (A, B) and 1 week after seeding (C). (D) Quantitative evaluation of cell numbers. Column 1: cells cultured for 1 day Column 2: cells cultured for 1 week. ${ }^{*} p<0.01$. These cells are ultra structurally similar to macrophages with a cytoplasm filled with electron-dense granules (arrows in E), are Giemsa positive cells (F) and immuno-stained with CD45 (G), CD68 (H) and Hm AIF-1 (J) antibodies, markers of macrophages cells. (L) Negative control in which the primary antibody was omitted. These cells show also a weak positivity to Thioflavin-S staining (yellow in M). Nuclei are counterstained with DAPI (blue in G-M). Bars in A, C: $50 \mu \mathrm{m}$; bars in B, D, F-M: $10 \mu \mathrm{m}$; bar in E: $2 \mu \mathrm{m}$.

cells were counted after one week from seeding. As shown by statistical analysis the number of cells increased by 2.5 times (Figure 3D). Trypan blue exclusion demonstrated that these cells were still viable (data not shown), exhibited the same morphological aspects described for the cells within the MG in vivo (Figure 3E), were positive for May Grunwald Giemsa differential staining (Figure 3F) and expressed the leukocyte-specific marker CD45 (Figure 3G), and the macrophage markers CD68 (Figure 3H) and HmAIF-1 (Figure 3I). No signal was visible in negative control sample (Figure $3 \mathrm{~L}$ ). Moreover these cells showed a weak positivity for Thioflavin-S dye (Figure 3M).

After obtaining primary culture of macrophages, we evaluated their ability to produce amyloid fibrils after MWCNTs exposure (Figure $4 \mathrm{~A}-4 \mathrm{X}$ ). $\mathrm{CD}^{+} 5^{+}$(Figure $4 \mathrm{~A}-4 \mathrm{~F}$ ) and $\mathrm{HmAIF}^{+} \mathrm{1}^{+}$(Figure 4G$4 \mathrm{~L})$ macrophages incubated for $24 \mathrm{~h}$ with increasing concentrations of MWCNTs $(2.5,5,10,25,50$ and $100 \mu \mathrm{g} / \mathrm{ml})$ were highly positive for Thioflavin-S staining. The amyloid fibrils stained in yellow (Figure $4 \mathrm{M}-4 \mathrm{R}$ ) were accumulated in large dilated reticulum cisternae filled with spatially organized fibrillar material, as visible by ultrastructural analysis at TEM (Figure $4 \mathrm{U}$ ) and then released in the extracellular environment (Figure 4T). Combined fluorescence/transmission images clearly showed that $\mathrm{HmAIF}-1^{+} / \mathrm{CD} 45^{+}$macrophage cells (Figure $4 \mathrm{~V}$ and $4 \mathrm{~W}$ ), strictly associated to MWCNTs aggregates, produce amyloid fibrils Thioflavin- $\mathrm{S}^{+}$(Figure $4 \mathrm{~S}$ and $4 \mathrm{~T}$ ).

\section{Discussion}

The contamination of water by various different pollutants is a serious problem worldwide in terms of human health and agriculture. MWCNTs are currently attracting intense research efforts because of their unique properties, which make them suitable for many industrial developments, applications in biomedicine, nanoelectronics, mechanical engineering, personal care products and textiles. MWCNTs are currently also produced and used in industry on a large-scale, increasing the risk for a widespread human and environmental exposure. Data in literature [21] have demonstrated that these nanoparticles have a very long half-life in vivo, could affect cellular functions at molecular levels and are capable of penetrating physiological barriers to reach vital organs inducing chronic inflammation, which it is often associated with insurgence of cancer. The physical dimensions and the biopersistence of MWCNTs were found to be similar to asbestos, and they indeed have revealed asbestos-like pathogenicity [22,23]. In order to avoid making the same mistakes, more research must be done on these new and emerging products in the market to provide a complete understanding of biological properties of MWCNTs, including uptake, distribution, intracellular trajectory, interactions with the immune system.

We have recently observed that in vivo treatment of $H$. medicinalis with water dispersed MWCNTs induce toxic effect also at low concentrations and after a short time of exposure. The 
Citation: Girardello R, Drago F, de Eguileor M, Valvassori R, Vizioli J, et al. (2015) Cytokine Impregnated Biomatrix: A New Tool to Study Multi-Wall Carbon Nanotubes Effects on Invertebrate Immune Cells. J Nanomed Nanotechnol 6: 323. doi:10.4172/2157-7439.1000323

Page 6 of 8

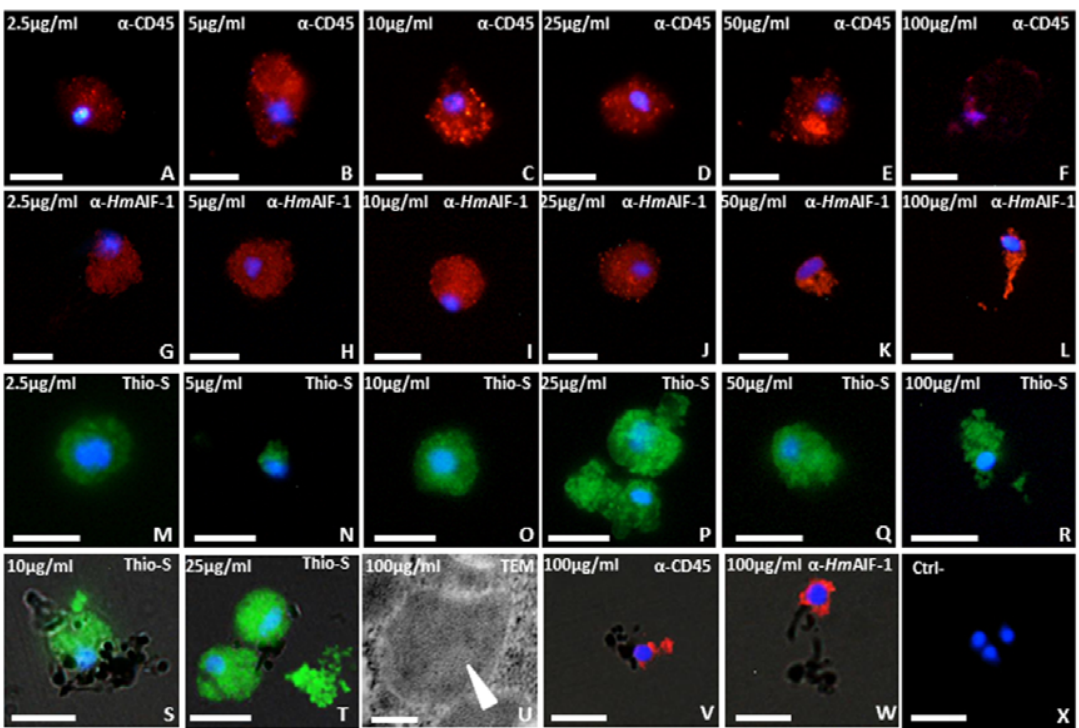

Figure 4: Effects of MWCNTs treatment on cultured macrophages. Cells extracted from MG after 1 week from injection and treated for $24 \mathrm{~h}$ with increasing concentration of MWCNTs $(2.5,5,10,25,50$ and $100 \mu \mathrm{g} / \mathrm{ml}$ ) are CD45 (red in A-F), Hm AIF-1+ (red in G-L) and show an increasing positivity for Thioflavin-S staining (yellow in M-R). Combined fluorescence/transmission (S, T) and TEM images clearly show the amyloid fibril deposition (yellow in S, T) and organized fibrillar material (arrowhead in $\mathrm{U}$ ) by CD45+/Hm AIF-1+ macrophages (V-W) strictly associated with MWCNTs aggregates. Nuclei in blue are stained with DAPI. No signal is detected in negative control experiments $(X)$. Bars in A-T, V-X: $10 \mu \mathrm{m}$; bar in U: $500 \mathrm{~nm}$

uptake of MWCNTs by leeches is associated with the induction of an inflammatory process, inducing a massive angiogenesis and migration of $\mathrm{CD}_{4} 5^{+}$and $\mathrm{CD} 8^{+}$macrophages throughout the animal body wall (Unpublished data, submitted to Plosone). These results suggest a novel entry mechanisms and toxicity profiles of MWCNTs, in fact, immunity is an essential function to retain organism's well-being, and represents a sensitive physiological indicator that may be affected even at low concentrations of nanomaterials exposure [24].

In order to better understand this point, we focused our attention on leech macrophages observing the behaviour of these cells in vivo and in vitro toward the nanomaterial. For this purpose we use a consolidated experimental approach based on a novel developed assay that allows the isolation of a specific cell population using the biomatrix Matrigel injected in the body wall of the leech [16-18].

We observe that aggregates of nanotubes are able to induce the migration of macrophages into the MG sponges. To increase the number of recruited cells in MG, allowing us to better analyze the internalization process of this nanomaterial, we loaded MG with both MWCNTs and the cytokine rHmAIF-1, which has been recently demonstrated to be a powerful chemo attractant for leech macrophages $[25,26]$. One week from injection, rHmAIF-1 invokes within the Matrigel a larger number of cells which are positive for the markers CD45, CD68 and HmAIF-1 which has been already demonstrated to be expressed by the monocyte/macrophage lineage both in leech and in vertebrates $[14,26-28]$. Ultrastructural analysis at TEM revealed that in leech macrophages MWCNTs are both internalized in vesicles and freely dispersed in the cytoplasm.

These data suggest that curled MWCNTs are internalized by phagocytosis or during the process of matrix degradation, while straight and rigid MWNCTs seem to be able to pierce cell membranes during cells migration and are then found free in the cytosol. These findings corroborate the observations of other authors on vertebrate macrophages [29-31]. Moreover, since recent experimental studies show that carbon nanotubes influence the aggregation process of proteins associated with neurodegenerative diseases like amyloid fibril production, we evaluate, by using the colorimetric methods of Thioflavin-S [32], the MWCNTs ability to induce amyloid deposits in correspondence of MWCNTs/macrophages associations [20]. Several studies demonstrated in fact that nanoparticles that enter cells by diffusing through cell membranes or by active uptake, such as endocytosis [33], cause toxic effects such as the formation of reactive oxygen species (ROS) [5,8,34]. Oxidative stress can induce proteins to adopt an insoluble beta-pleated sheet conformation [35], and according to numerous authors [36-40] oxidative damage appears to be the earliest events preceding amyloid fibril formation. Thus, we evaluate the amyloid fibril production from macrophage in relation to MWCNTs exposure. As expected, we found a massive accumulation of fibrils Thioflavin- $\mathrm{S}^{+}$, associated to the macrophages forming a scaffold around the MWCNTs aggregates. The presence of these fibrils in MWCNTs and rHmAIF-1 supplemented MG confirms the strong reaction of macrophages to the nanomaterial.

Starting from these results, the next target of our work was to obtain in vitro expansion of macrophages primary leech cells that could be used as a sensitive method to evaluate the presence of the nanomaterial in contaminated water. We used the biopolymer MG supplemented with $\mathrm{rHm}$-AIF-1 as a vector to isolate these cells in vivo and then culture them obtaining an in vitro expansion of macrophages primary leech cells. Seven days after seeding cells cultured in a normal medium expressed the same specific macrophage markers (CD45, CD68, HmAIF-1) as that already observed in vivo. The ability of MWCNTs to induce amyloid fibrils generation were assessed by measuring Thioflavin-S fluorescence as a reporter of amyloid fibrils generation. As compared with control unexposed cells, a significant increase in Thioflavin-S staining was observed in exposed macrophage cell in concentration independent manner and the amyloid fibrils formed 
Citation: Girardello R, Drago F, de Eguileor M, Valvassori R, Vizioli J, et al. (2015) Cytokine Impregnated Biomatrix: A New Tool to Study Multi-Wall Carbon Nanotubes Effects on Invertebrate Immune Cells. J Nanomed Nanotechnol 6: 323. doi:10.4172/2157-7439.1000323

a scaffold around the MWCNTs aggregates, indicating that amyloid deposition might be a barrier to contain non-self-material.

Recent data on murine model [20] showed inflammatory responses generated by commercially available nanotubes that are inhaled. Moreover, literature is beginning to show data concerning the potential risks to public health and the aquatic environment when these nanomaterials are dispersed in water. Our data on leeches confirmed the toxicological effects of nanotubes. The use of annelids to implement the data so far produced is in order to demonstrate the reliability and reproducibility of the new model in respect to those commonly utilized by eco-toxicologists. In fact, unlike other invertebrates, $H$. medicinalis use the same strategies and the same molecules of vertebrates in response to different stimuli. For these reasons, it is critical to investigate the question of the nanomaterial safety utilizing new models and methods for assessing the environmental risks of these possible toxic particles.

The data produced by this project will be a cornerstone in determining the potential toxicity of MWCNTs and the eventual precautions needed in the wastewater discharge. Our results also provide critical information to regulatory agencies and industry to determine the need for monitoring and regulation regarding MWCNTs.

\section{Acknowledgement}

Dr. Rossana Girardello is a student of the Ph.D. program in Biotechnology, Biosciences and Surgical Technologies, School in Biological and Medical Sciences, University of Insubria. This work was supported by CARIPLO foundation 2012 to VR. The authors wish to thank the Centro Grandi Attrezzature (CGA) of the University of Insubria.

\section{References}

1. Ji Z, Zhang D, Li L, Shen X, Deng X, et al. (2009) The hepatotoxicity of multiwalled carbon nanotubes in mice. Nanotechnology 20: 445101.

2. Theron J, Walker JA, Cloete TE (2008) Nanotechnology and water treatment: Applications and emerging opportunities. Crit Rev Microbiol 34: 43-69.

3. Du J, Wang S, You H, Zhao X (2013) Understanding the toxicity of carbon nanotubes in the environment is crucial to the control of nanomaterials in producing and processing and the assessment of health risk for human: A review. Environ Toxicol Pharmacol 36: 451-462.

4. Saria R, Mouchet F, Perrault A, Flahaut E, Laplanche C, et al. (2014) Shor term exposure to multi-walled carbon nanotubes induce oxidative stress and DNA damage in Xenopus laevis tadpoles. Ecotoxicol Environ Saf 107: 22-29.

5. Pereira MM, Mouton L, Yepremian C, Coute A, Lo J, et al. (2014) Ecotoxicological effects of carbon nanotubes and cellulose nanofibers in Chlorella vulgaris. J Nanobiotechnology 12: 1-13.

6. Velzeboer I, Peeters ETHM, Koelmans AA (2013) Multiwalled carbon nanotubes at environmentally relevant concentrations affect the composition of benthic communities. Environ Sci Technol 47: 7475-7482.

7. Teow Y, Asharani PV, Hande MP, Valiyaveettil S (2011) Health impact and safety of engineered nanomaterials. Chem Commun (Camb) 47: 7025-7038.

8. Funahashi S, Okazaki Y, Ito D, Asakawa A, Nagai H, et al. (2015) Asbestos and multi-walled carbon nanotubes generate distinct oxidative responses in inflammatory cells. J Clin Biochem Nutr 56: 111-117.

9. Dörger M, Münzing S, Allmeling AM, Messmer K, Krombach F (2001) Differential responses of rat alveolar and peritoneal macrophages to man-made vitreous fibers in vitro. Environ Res 85: 207-214.

10. Boyles MSP, Young L, Brown DM, MacCalman L, Cowie H, et al. (2015) Multiwalled carbon nanotube induced frustrated phagocytosis, cytotoxicity and proinflammatory conditions in macrophages are length dependent and greater than that of asbestos. Toxicol Vitr 29: 1513-1528.

11. De Eguileor M, Tettamanti G, Grimaldi A, Congiu T, Ferrarese R, et al. (2003) Leeches: Immune response, angiogenesis and biomedical applications. Curr Pharm Des 9: 133-147.
12. De Eguileor M, Grimaldi A, Tettamanti G, Valvassori R, Cooper EL, et al. (2000) Different types of response to foreign antigens by leech leukocytes. Tissue Cell 32: 40-48.

13. Grimaldi A, Tettamanti G, Rinaldi L, Perletti G, Valvassori R, et al. (2004) Role of cathepsin B in leech wound healing. ISJ: 38-46.

14. Grimaldi A, Tettamanti G, Perletti G, Valvassori R, de Eguileor M (2006) Hematopoietic cell formation in leech wound healing. Curr Pharm Des 12 3033-3041.

15. Tettamanti G, Malagoli D, Benelli R, Albini A, Grimaldi A, et al. (2006) Growth factors and chemokines: A comparative functional approach between invertebrates and vertebrates. Curr Med Chem 13: 2737-2750.

16. Grimaldi A, Bianchi C, Greco G, Tettamanti G, Noonan DM, et al. (2008) In vivo isolation and characterization of stem cells with diverse phenotypes using growth factor impregnated biomatrices. PLoS One 3.

17. Grimaldi A, Banfi S, Gerosa L, Tettamanti G, Noonan DM, et al. (2009) Identification, isolation and expansion of myoendothelial cells involved in leech muscle regeneration. PLoS One 4.

18. Grimaldi A, Banfi S, Vizioli J, Tettamanti G, Noonan DM, et al. (2011) Cytokine Loaded Biopolymers as a Novel Strategy to Study Stem Cells during WoundHealing Processes. Macromol Biosci 11: 1008-1019.

19. Kibbey MC (1994) Maintenance of the EHS sarcoma and Matrigel preparation $\mathrm{J}$ Tissue Cult Methods 16: 227-230.

20. Albini A, Pagani A, Pulze L, Bruno APE (2015) Environmental impact of multiwall carbon nanotubes in a novel model of exposure: Systemic distribution macrophage accumulation and amyloid deposition. Int J Nanotechnol in press.

21. Oberdörster G, Oberdörster E, Oberdörster J (2005) Nanotoxicology: An emerging discipline evolving from studies of ultrafine particles. Environ Health Perspect 113: 823-839.

22. Poland CA, Duffin R, Kinloch I, Maynard A, Wallace W a H, et al. (2008) Carbon nanotubes introduced into the abdominal cavity of mice show asbestos-like pathogenicity in a pilot study. Nat Nanotechnol 3: 423-428.

23. Murphy FA, Schinwald A, Poland CA, Donaldson K (2012) The mechanism of pleural inflammation by long carbon nanotubes: Interaction of long fibres with macrophages stimulates them to amplify pro-inflammatory responses in mesothelial cells. Part Fibre Toxicol 9: 8 .

24. Hayashi Y, Engelmann P (2013) Earthworm's immunity in the nanomaterial world : New room , future challenges. Invertebr Surviv J 10: 69-76.

25. Drago F, Sautière PE, Le Marrec-Croq F, Accorsi A, Van Camp C, et al. (2014) Microglia of medicinal leech (Hirudo medicinalis) express a specific activation marker homologous to vertebrate ionized calcium-binding adapter molecule 1 (Iba1/alias aif-1). Dev Neurobiol 1

26. Schorn T, Drago F, Tettamanti G, Valvassori R, de Eguileor M, et al. (2015) Homolog of allograft inflammatory factor-1 induces macrophage migration during innate immune response in leech. Cell Tissue Res 359: 853-864.

27. Yang ZF, Ho DW, Lau CK, Lam CT, Lum CT, et al. (2005) Allograft inflammatory factor-1 (AIF-1) is crucial for the survival and pro-inflammatory activity of macrophages. Int Immunol 17: 1391-1397.

28. Tian Y, Kelemen SE, Autieri M V (2006) Inhibition of AlF-1 expression by constitutive siRNA expression reduces macrophage migration, proliferation, and signal transduction initiated by atherogenic stimuli. Am J Physiol Cell Physiol 290: C1083-C1091.

29. Pulskamp K, Diabaté S, Krug HF (2007) Carbon nanotubes show no sign of acute toxicity but induce intracellular reactive oxygen species in dependence on contaminants. Toxicol Lett 168: 58-74.

30. Di Giorgio ML, Bucchianico S Di, Ragnelli AM, Aimola P, Santucci S, et al. (2011) Effects of single and multi walled carbon nanotubes on macrophages: Cyto and genotoxicity and electron microscopy. Mutat Res - Genet Toxicol Environ Mutagen 722: 20-31.

31. Nagai H, Toyokuni S (2012) Differences and similarities between carbon nanotubes and asbestos fibers during mesothelial carcinogenesis: Shedding light on fiber entry mechanism. Cancer Sci 103: 1378-1390.

32. Grimaldi A, Girardello R, Malagoli D, Falabella P, Tettamanti G, et al. (2012) Amyloid / Melanin distinctive mark in invertebrate immunity. ISJ 9: 153-162.

33. Johnston HJ, Hutchison GR, Christensen FM, Aschberger K, Stone V (2009) 
Citation: Girardello R, Drago F, de Eguileor M, Valvassori R, Vizioli J, et al. (2015) Cytokine Impregnated Biomatrix: A New Tool to Study Multi-Wall Carbon Nanotubes Effects on Invertebrate Immune Cells. J Nanomed Nanotechnol 6: 323. doi:10.4172/2157-7439.1000323

Page 8 of 8

The biological mechanisms and physicochemical characteristics responsible for driving fullerene toxicity. Toxicol Sci 114: 162-182

34. Li N, Xia T, Nel A (2008) The Role of Oxidative Stress in Ambient Particulate Matter- induced Lung Diseases and Its Implications in the Toxicity of Engineered Nanoparticles. Free Radic Biol Med 44: 1689-1699.

35. Drake J, Link CD, Butterfield DA (2003) Oxidative stress precedes fibrillar deposition of Alzheimer's disease amyloid ??-peptide (1-42) in a transgenic Caenorhabditis elegans model. Neurobiol Aging 24: 415-420.

36. Christen Y (2000) Oxidative stress and Alzheimer disease 1, 2. 71.
37. Squier TC (2001) Oxidative stress and protein aggregation during biological aging. Exp Gerontol 36: 1539-1550.

38. Zhu X, Raina AK, Perry G, Smith M a. (2004) Alzheimer's disease: The two-hit hypothesis. Lancet Neurol 3: 219-226.

39. Chen Q, Ding Q, Keller JN (2005) The stationary phase model of aging in yeast for the study of oxidative stress and age-related neurodegeneration. Biogerontology 6: 1-13.

40. Yan HD, SS (2010) Mitochondrial permeability transition pore in Alzheimer's disease: Cyclophilin D and amyloid beta. Biochim Biophys Acta 1802: 198-204. 